\section{MS39-05 Indexing of multi-crystal snapshots collected with a broad bandpass beam}

Stef Smeets ${ }^{1}$, Catherine Dejoie ${ }^{1}$, Christian Baerlocher ${ }^{1}$, Lynne B. McCusker $^{1}$

\section{Laboratory of Crystallography, ETH Zurich, Switzerland}

email: stef.smeets@mat.ethz.ch

$\mathrm{X}$-FEL sources provide new opportunities for analyzing difficult crystal structures. Our interest is in organic/inorganic materials with unit cells much smaller than those of the macromolecular crystals that have been the focus of most X-FEL studies to date. We are developing a strategy for the collection and processing of such data [1], based on the broad-bandpass mode (4\% energy bandwidth) that will be provided at SwissFEL [2]. The crystals are destroyed by the extremely intense X-ray pulse, so only single snapshots of the sparse data can be recorded, and this makes indexing a challenge. The problems to be overcome are four-fold: (1) accurate orientations have to be retrieved from a single frame, (2) the wavelength associated with each diffraction spot is indeterminate, (3) the unit cells are small, so the number of observations per frame is limited, and (4) the orientations of multiple crystals should be determined from a single frame.

Experiments mimicking a $4 \%$-energy-bandpass mode were performed on SNBL at the ESRF on samples of the zeolite ZSM-5 (up to 15 crystals measured simultaneously), the mineral sanidine, and a cesium cyanoplatinate. We examined several existing methods for indexing these data, assuming the unit cell to be known a priori, but most of them proved to be ill-suited. Only a modified Laue-based approach could be applied successfully, but computation times became a limitation in the multi-crystal experiments. Therefore, a new strategy was developed to deal specifically with the data at hand. Candidate crystal orientations are generated either by searching for the indices that match the d-spacings and angles of two low-resolution non-collinear reflections, or with a brute force approach that tries 1.5 million roughly equally distributed rotation matrices that cover all possible crystal orientations. The idea is that only a good solution will index a large number of reflections. To index multiple patterns, the smallest subset of orientation matrices that indexed the largest number of reflections are to be identified. For each sample, the indexing routine performed admirably, and the orientations of up to 11 crystals could be determined from a single frame. While our algorithms were developed with SwissFEL in mind, they can be applied to any data collected in single snapshot mode with either monochromatic or broad-bandpass radiation.

[1] C. Dejoie et al., IUCrJ, 2015, accepted.

[2] B. D. Patterson et al., Chimia, 2014, 68, 73-78.

Keywords: X-FEL, indexing, broad bandpass beam, multi-crystal diffraction

\section{MS40. X-ray diffraction from \\ microsecond to femtosecond time range (including FELs)}

\author{
Chairs: Christian Betzel, Anton Barty
}

\section{MS40-01 Sample preparation and delivery for serial and time-resolved crystallography}

Dominik Oberthuer ${ }^{1,2}$

1. University of Hamburg, Luruper Chaussee 149, 22607 Hamburg, Germany

2. Center for Free-Electron Laser Science, Deutsches Elektronen-Synchrotron DESY, Notkestraße 85, 22607 Hamburg, Germany

\section{email: dominik.oberthuer@desy.de}

Serial crystallography methods at both X-ray free-electron lasers (Chapman, 2011) and third generation synchrotron sources (Gati, 2014; Stellato, 2014) are now well established, making it possible to determine the structures of proteins that only form very small crystals or that are extremely radiation sensitive. Serial crystallography methods are also very well suited for time-resolved experiments (Tenboer, 2014), making it possible to reveal the dynamic nature of biological macromolecules and their interactions at near-atomic resolution and on ultrafast timescales. In case of such experiments one deliberately strives to grow micro or sub-micron sized crystals of proteins that would otherwise form larger crystals as well. Small crystals not only allow for uniform laser excitation of all unit-cells in the X-ray beam, but possibly for future mix-and-diffuse studies of reactions that cannot be photo-induced. Size homogeneity of the crystals and, since the amount of sample is limited, a high yield of crystals and optimized sample delivery methods are very important in this regard. Here promising new crystallization methods will be shown, as well as a pipeline for crystal characterization prior to the crystallographic experiment and an overview on strategies to reduce sample consumption. Chapman, H. N., (2011) Femtosecond X-ray protein nanocrystallography. Nature 470, 73-77 Gati, C., (2014) Serial crystallography onin vivogrown microcrystals using synchrotron radiation. IUCrJ 1 Stellato, F., (2014) Room-temperature macromolecular serial crystallography using synchrotron radiation. IUCrJ 1, 204-212 Tenboer, J., (2014) Time-resolved serial crystallography captures high-resolution intermediates of photoactive yellow protein. Science 346, 1242-1246

Keywords: Serial Crystallography, FEL, Crystallization, Methods 